\title{
Phase II Trial
}

National Cancer Institute

\section{Source}

National Cancer Institute. Phase II Trial. NCI Thesaurus. Code C15601.

A clinical research protocol designed to study a biomedical or behavioral intervention in a

larger group of people (several hundred), to evaluate the drug's effectiveness for a

particular indication in patients with the disease or condition under study, and to

determine the common short-term side effects and risks associated with the intervention. 